\title{
Controllability of Spacecraft Systems in a Central Gravitational Field ${ }^{1}$
}

\author{
Kuang-Yow Lian ${ }^{t}$, Li-Sheng Wang ${ }^{\dagger}$, and Li-Chen Fu ${ }^{\dagger}$
}

Department of Electrical Engineering ${ }^{\dagger}$

Institute of Applied Mechanicst

National Taiwan University, Taipei, Taiwan, R.O.C.

Abstract

The natural configuration space for spacecraft systems in a central gravitational field can be modeled by $S O(3) \times \mathbb{R}^{3}$, where the $S$ pecial Orthogonal Group $S O(3)$ represents the attitude dynamics and $\mathbb{R}^{3}$ is for the orbital dynamics. While the attitude dynamics of the spacecraft system is affected by the orbital elements through the well-known gravity-gradient torque, it has not been noted before, to our knowledge, that the effects of attitude-orbit coupling can be used to change orbital that the effects of attitude-orbit coupling can be used to change orbital
motions by controlling the attitude. This controllability property is the main issue of this work. We shall prove that the control systems for spacecraft with reaction wheels or gas jets as attitude controllers are controllable.

\section{Introduction}

For a spacecraft system moving in a central gravitational field, the gas jets, or thrusters, acting directly on the orbital elements, are commonly used in orbital maneuvers. Typically, the satellite is modeled as a point mass and the orbits are simply Keplerian orbits. However, if a rigid body with finite extent is chosen to model the spacecraft, the orbital motion will be then coupled with the attitude dynamics. The Haniltonian structure of such system and the effects of this coupling have been discussed in $[28,29,31,30]$. With this coupling, it was then suspected that controlling the attitude motion may eventually affect the orbital motion. This then leads to the notion of which control only the attitude. This idea notivated our work in this paper.

The natural configuration manifold for spacecraft systems in a central gravitational field can be modeled as $S O(3) \times \mathbb{R}^{3}$, or abbreviated as $S E(3)$. The Special Orthogonal Group $S O(3)$ refers to the configuration space of attitude dynamics, while $\mathbb{R}^{3}$ is for the configuration space of orbital (or translational) dynamics. How to control the attitude motion and orbital motion (efficiently) during maneuver keeps being an important issue in spacecraft controller design. More specifically, various control strategies have pacecraft controller design. More specifically, various control strategies have
been used in controlling the attitude. These inchude thrusters (gas jets), magbeen used in controlling the attitude. These inchude thrusters (gas jets), maggyros, momentum wheels, and reaction wheels, see, e.g. [32, 33]. Concerning the efficiency, power, and accuracy of the control systems, an effective 3-axis stabilization scheme may use reaction wheels for attitude control with the momentum dumping through the thrusters. On the other hand, as described in the precious paragraph, orbital controls are mainly performed through gas jets. In previous design of such controllers, the gravity-gradient torque is
sometimes included as disturbance torque or is used to assist the controller design. This gravity-gradient torque techmique exhibits one of the coupling effects on the attitude from the orbital motion. However, as will be demonstrated later, this coupling also appears in orbital dynamical equations. Accordingly, how to assess this effect on orbital dynamics and how to properly use them to assist orbital control become an interesting question.

The controllability problem has attracted nany investigators for its important role in control theory and applications. For a general nonlinear system the sufficient and necessary conditions, i.e. the Lie algebra rank condition, for local accessibility (or weak controllability) for analytical systems was addressed in many places, such as $[25,14,10]$. In order to guarantee that a point $p$ is in the interior of the reachable set from $p$ for arbitrary small time, the notion of small time local controllability was introduced in [16]. Many sufficient conditions for the small time local controllability have been obtained in $[6,11,9]$. In [23], Sussman proved a general theorem accommodating these conditions by applying the idea of action of Lie groups on the state-space of the dynamical system. For general nonlinear systems the concept of (global) controllability is very sophisticated, $\mathrm{cf}$. $[5,24]$. Although there are no com-
pletely satisfactory criteria has been developed, there are fruitful results for pletely satisfactory criteria has been developed, there are fruitful results for the controllability can be established for Poisson stable systems satisfying the controllability can be established for Poisson stable systems satisfying requires the drift vector field (i.e. the vector field with no controls) having the Poisson stability property, can be founded in [13], and [4]. These result-

'This work was partially supported by National Science Council, flepublic of Chima, under grant NSC 82-0208-M-002-028, NSC: 82-0422-E-002-045 and NSC 82-F-SP-002-03

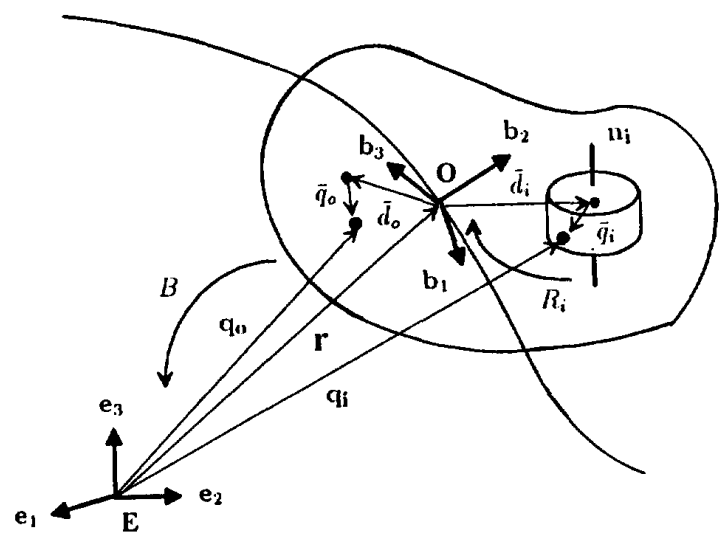

Figure 1: Spacecrraft with Reaction Wheels in a Central Force Field

$s$ have been applied in [8] to established the controllability property of the spacecraft system in which only attitude dynamics were considered. In this paper, a slight generalization of the controllability theory mentioned above will be constructed. The requirement of Poisson stability will be replaced by a weaker condition, i.e. weakly positively Poisson stability. This theorem will be then directly used to prove the controllability of the spacecraft systems, which include attitude-orbit coupling, controlled by using reaction wheels or gas jets in only attitude controllers. This is one of the main contributions of this work.

In fact, we shall show that for a dynamical system with weakly positively Poisson stable drift vector field, the Lie algebra rank condition will imply controllability. For the spacecraft system under consideration, its intrinsic Hamiltonian structure can be utilized to prove the weakly positively Poisson stability by applying Poincare recurrence theorem. On the other hand, the
work of checking the Lie algebra rank condition can be carried out with the aid of the general formula for the Lie bracket computation in $T^{*} T\left(S O(3)^{m} \times\right.$ $\mathbb{I R})^{n}$ developed in [17][18]. With these tools, the controllability can be then concluded.

The rest of this paper is organized as follows. A quick review of equations

The rest of this paper is organized as follows. A quick review of equations
of motion for the spacecraft systems is given in Section 2. Formal definiof motion for the spacecraft systems is given in Section 2 . Formal definitions of controllability and weakly positive Poisson stability will be given in putation on jet bundles of $S O(3)$ are then used to prove the controliability putation on jet bundles of $S O(3)$ are then used to prove the controllability of spacecraft systems in Section 4. In order to verify the Lie algebra rank condition, proper approximations for the gravity term are adopted. We shal1 prove that for some spacecraft without any axis-symmetry and satisfying some conditions, the controllability of the coupled system by using gas jets or reaction wheels in attitude controiler can be concluded. Consequently, the coupling effects of at

motion for a spacecraft. However it has been noted, of [22], that the center of gravity of the assembly is different from the center of mass and the former may change its location is diferent from the center of mass and the former may change its location with respect to different attitudes. This is one place where the coupling effects appear. From this observation, it is then natural that controlling the attitude may lead to some change of orbital motion. Accordingly, the controllability
results are also physically appealing.

\section{System Description} Consider a system consisting of a rigid body, or platform, with some sym-
metric rotors, moving in a central gravitational fieid, as depicted in Figure 1 . Let $E$ denote the center of a gravitational field (e.g. generated by the Earth) with gravitational constant $\mu$. The system of the platform of mass $m_{0}$ and $l$
. 
rotors of masses $m_{i}, i=1, \cdots, l$, moves around the center $E$. The total mass of the assembly $E$ will be denoted by $m$. Let $O$ denote the center of mass of the assembly. We attach an inertial reference frame $\left\{\mathbf{e}_{1}, \mathbf{e}_{2}, \mathbf{e}_{3}\right\}$ to $E$ and body reference frame $\left\{b_{1}, b_{2}, b_{3}\right\}$ to $O$, respectively. The radius vector from $E$ to $O$ is represented by $\mathbf{r} \in \mathbb{R}^{3}$ in inertial frame.

The attitude of the assembly can be modeled by an element $B \in S O(3)$, which represents the rotation of the body frame relative to the inertial frame. The special orthogonal group or rotation group $S O(3)$, is defined to be the

$$
S O(3)=\left\{B \in \mathbb{R}^{3 \times 3} \mid B^{\top} B=1, \operatorname{det}(B)=1\right\},
$$

which is a subset of $3 \times 3$ nonsingular matrices. Here, and in what follows, 1 denotes the identity matrix of the appropriate dimension.

Let $s o(3)$ denote the space of $3 \times 3$ skew-symmetric matrices. A natural isomorphism between $\mathbb{R}^{3}$ and so(3) can be constructed as, with the operator

$$
\left(\begin{array}{l}
\widehat{a_{1}} \\
a_{2} \\
a_{3}
\end{array}\right)=\left(\begin{array}{ccc}
0 & -a_{3} & a_{2} \\
a_{3} & 0 & -a_{1} \\
-a_{2} & a_{1} & 0
\end{array}\right)
$$

The kinematic equation for a rotation matrix can be written as $\dot{B}=B \hat{\Omega}$ where $\Omega$ is the instantaneous body angular velacity of the assembly.

For the $i$-th rotor, the location of its center of mass and orientation of $i$ axis of symmetry will be denoted by $\bar{d}_{i}$ and a unit vector $\boldsymbol{n}_{i}$, respectivel both in body frame. The location of the center of mass of the platform will be denoted by $\bar{d}_{0}$ in body frame. The motion of $i$-th rotor relative to the platform can be described by an angle $\theta_{i}$, or equivalently, by an element in the circle group $S^{1}$. As a consequence, the configuration space of the assembly can be modeled as $S O(3) \times T^{n} \times \mathbb{R}^{3}$.

Let $\mathbf{I}_{o}$ and $\mathbf{I}_{i}$ denote, respectively, the moments of inertial tensor of the platform and of the $i$-th rotor about their centers of masse. The equation of motion for the spacecraft system with 1 reaction wheels in a central gravitational field can be derived by using the Euler-Lagrange method [17] or Newton-Euler method [30] and are expressed as follows,

$$
\begin{aligned}
\dot{B} & =B \hat{\Omega}, \\
\dot{\mathbf{S}}+\dot{\eta} & =-\Omega \times(\mathbf{I} \Omega+\eta)+\int_{\mathcal{B}} \frac{\mu\left(B^{\mathrm{T}} \mathbf{r} \times \bar{q}\right)}{|\mathbf{r}+B \bar{q}|^{3}} d m(\bar{q}), \\
I_{i} \dot{\Omega}^{\top} \mathbf{n}_{i}+I_{i} \ddot{\theta_{i}} & =\tau_{i}, \quad \forall i=1, \ldots, l, \\
m \ddot{\mathbf{r}} & =-\int_{\mathcal{B}} \frac{\mu(x+B \bar{q})}{|\mathbf{r}+B \bar{q}|^{3}} d m(\bar{q}),
\end{aligned}
$$

where $\mathbf{I}=\sum_{i=0}^{l}\left(\mathbf{I}_{i}+m_{i} \hat{\vec{d}}_{i}^{\top} \hat{\vec{d}}_{i}\right), I_{i}$ stands for the eigenvalue of $\mathbf{I}_{i}$ along the eigenvector $\mathbf{n}_{i}, \tau_{i}$ is the torque generated by the acturator acting on the $i$-th rotor, and $\eta$ denotes the total angular momentum of rotors relative to the
body,

$$
\eta=\sum_{i=1}^{l} \dot{\theta}_{i} \mathbf{I}_{i} \mathbf{n}_{i}
$$

\section{Poisson Stability and Controllability}

Before answering the controllability problem for the spacecraft system, we need to give formal definitions and obtain some criteria to suit our needs. Consider a smooth vector field $X$ on a smooth connected manifold $M$. Let

$$
\phi^{\mathbf{x}}: \mathbb{R} \times M \rightarrow M ; \quad(t, p) \mapsto \phi_{1}^{\mathbf{x}}(p) .
$$

Assume that $X$ is complete or $\phi_{1}^{x}(p)$ is defined for all $t \geq 0$. A point in $M$ is called positively Poisson stable for $X$ if for all $T>0$ and for any neighborhood $U_{p}$ of $p$, there exists a time $t>T$, such that

$$
\phi_{t}^{\times}(p) \in U_{p}
$$

The vector field $X$ is called positively Poisson stable if the set of positively Poisson stable points for $X$ is dense in $M$.

A point $p \in M$ is called a nonwandering point of $X$ if for all $T>0$, and for any neighborhood $U_{p}$ of $p$, there exists a time $t>T$ such that

$$
\phi_{t}^{\times}\left(U_{p}\right) \cap U_{p} \neq \emptyset,
$$

where $\phi_{t}^{\mathrm{x}}\left(U_{p}\right)=\left\{\phi_{t}^{\mathrm{x}}(q): q \in U_{p}\right\}$. A positively Poisson stable point is necessarily a nonwandering point. Let. $\Gamma_{x}$ denote the nonwandering set, which is defined to be the set of all nonwandering points of $X$. We have the following lemma. Lemma 1 The nonwandering set of a positively Poisson stable vector field

Proof: Let $S$ denote the set of positively Poisson stable points for $X$. By definition, the closure of $S$ el( $\left.S^{\prime}\right)$, is equal to $M$. That is for any $p \in M$ given $U_{p}$, a neighborhood of $p$, we have $U_{p} \cap S \neq 0$. This implies that there exists a positively Poisson stable point $q$. Let $U_{q} \in U_{p}$, be a neighborhood of $q$. We have $\forall T>0, \exists t>T, \phi_{t}^{x}(q) \in U_{q}$. Hence $\phi_{t}^{x}(q) \in U_{p}$, which implies
$\phi_{i}^{x}\left(U_{p}\right) \cap U_{p} \neq 0$. Thus $p$ is a nonwandering point. Since $p$ is arbitrary, we

From this lemma, the conditions for a vector field with the whole manifold $M$ being its nonwandering set are weaker than the conditions for it being positively Poisson stable. Accordingly, a vector field will be called weakly positively Poisson stable if its nonwandering set is $M$

Recall that in classical mechanics Liouville theorem tells us that the flow enerated by a time-independent Hamiltonian system is volume-preserving. On the other hand, Poincaré recurrence theorem proved that for a volumepreserving, bijective, continuous map $g$ on a bounded domain $D \subset \mathbb{R}^{n}$, every neighborliood $U$ of each point in $D$ contains a point $q$ which returns to $U$ after repeated application of the mapping, i.e., $g^{n}(q) \in U$ for some for some positive integer $n$. By combining the above observations, of. $[3,27]$, we have the following theorem:

Theorem 1 A time-independent Hamiltonian vector field on a manifold which is bounded is weakly positively Poisson stable.

A dynamical polysystem, c.f. [19], is defined to be a collection of comp]lete vector fields on $M$, which can be denoted by $\mathcal{F}=\left\{X_{\alpha}, \alpha \in I\right\}$ where $I$ is an index set. An integral curve of $\mathcal{F}$ is a mapping $\Phi:\left[t_{0}, t_{f}\right] \rightarrow M$ such that there exist vector fields, $X_{1}, \ldots, X_{k}$ in $\mathcal{F}$ and $t_{o}<t_{1}<\cdots<t_{k}=t_{f}$, with the property that the restriction of $\Phi$ to $\left[t_{i-1}, t_{i}\right]$ is an integral curve of $X_{i}$ for each $i=1,2, \ldots, k$.

Let $U$ be an open connected subset of $M$ and $T \geq 0$. A point $q \in U$ 位 integral curve $\Phi(t) \in U$ for all $t \in[0, T]$ such that $\Phi(0)=p$ and $\Phi(T)=q$. The set of all locally $\mathcal{F}$-reachable point from $p$ at time $T$ will be denoted by $\mathbf{R}^{U}(\mathcal{F}, T, p)$. Let $\mathbf{R}_{T}^{U}(\mathcal{F}, p)$ denote $U_{0 \leq t \leq T} \mathbf{R}^{U}(\mathcal{F}, t, p)$

Definition 1 A dynamical polysystem $\mathcal{F}$ is said to be locally accessible if restricted to every open connected subset $U$ of $M$, for all $T>0, \mathrm{R}_{T}^{U}(\mathcal{F}, p)$ has a nonempty interior subset for every $p \in U$. It is said to be accessible if for all $T>0, \operatorname{R}_{T}^{M}(\mathcal{F}, p)$ has a nonempty interior subset for every $p \in M$. $\quad \square$

The local accessibility, which is sometimes called locally weak controllabil ity, means that for every $p, q \in U$, any $T>0$, there exists an integral curve in $U$ steering $p$ to $q$ or (not necessary and) $q$ to $p$ within the interval $(0, T)$.

Definition 2 A dynamical polysystem $\mathcal{F}$ is said to be locally controllable if restricted to every open connected subset $U$ of $M$ and for every $p \in U$ $\mathrm{R}_{T}^{U}(\mathcal{F}, p)=M$. It is said to be controllable if $\mathrm{R}_{T}^{M}(\mathcal{F}, p)=M$ for every $p \in M$

Accordingly, local controllability (accessibility) implies controllability (accessibility). However, it is noteworthy that, in contrast with $[10,15,7,20]$ with the above definitions, (locally) controllable does not imply (locally) accessible. This is because that for accessibility, the nonempty interior property cessible. This is because that for accessibility, the nonempty interior property
must be satisfied for all $T>0$ while for controllability, we only ask for some $T>0, \mathrm{R}_{T}^{L}(\mathcal{F}, p)=M$. For the brevity of notations, in the sequel, we shall adopt the convention that if $T$ is suppressed, $\mathrm{R}^{U}(\mathcal{F}, p)$ is to be understood as $\mathbf{R}_{T}^{U}(\mathcal{F}, p)$ for some $T>0$, while if $U$ is suppressed, $U=M$, e.g., $\operatorname{R}(\mathcal{F}, T, p)$ is to be understood as $\mathbf{R}^{M}(\mathcal{F}, T, p)$

The necessary and sufficient conditions for local accessibility are given by the following theorem:

Theorem 2 [10] Let $\mathcal{L}(\mathcal{F})$ denote the smallest Lie algebra in $\mathcal{X}(M)$ that contains $\mathcal{F}$. The dynamical polysystem $\mathcal{F}$ is locally accessible if (and only if for the analytical case

$$
\operatorname{span}((\mathcal{L}(\mathcal{F}))(p))=T_{p} M
$$

for all $p \in M$

In the following, condition (3) will be called the Lie algebra rank condition. It has also been stated in [19] that for the analytical case, the polysystem $\mathcal{F}$ which is controllable must satisfy the Lie algebra rank condition. Therefore, (local) controllability will imply local accessibility for analytic vector fields.

Next we consider a general nonlinear control system:

$$
\dot{x}=f(x)+\sum_{i=1}^{j} g_{i}(x) u_{i} ; x \in M,
$$

where $f, g_{1}, \ldots, g_{j}$ are analytic vector fields on $M$, and the control $u: \mathbb{R}^{+} \rightarrow$ $u$, a bounded subset of $\mathbb{R}^{j}$, is a measurable function. Let $\boldsymbol{X}^{a}(M)$ denote the set of all analytical vector fields on $M$. Let $\mathcal{F} \equiv\left\{f+\sum_{i=1}^{j} g_{i} u_{i} \mid u \in U\right\}$ define a dynamical polysystem. An integral curve of $\mathcal{F}$ essentially corresponds to a trajectory of (4) witl piecewise constant inputs. The smallest Lie algebra in $\mathcal{X}^{a}(M)$ that contains $\mathcal{F}$ is equivalent to the Lie algebra genterated by $\left\{f, g_{1}, \ldots, g_{j}\right\}$

It was proved in [21] that for $f=0$, and $\mathcal{F}$ defined above being symmetric, i.e., for any $X \in \mathcal{F}$, also $-X \in \mathcal{F}$, then the locally accessibility implies controllability. However the control systems we are dealing with are symmetric. The method of analyzing the controllability of with are not system in the subsequent sections is based on the following controllability theorem: 

Theorem 3 Suppose that $f$ is a weakly positively Poisson vector field. The
system (4) is controllable if and only if it is locally accessible.

Before proving the theorem, we introduce the following lemma.

Lemma 2 [12] Let $\mathcal{F}$ be a dynamical polysystem. Then

$$
\mathrm{cl}(\mathrm{R}(\mathcal{F}, p))=\mathrm{cl}(\mathrm{R}(\operatorname{conv}(\mathcal{F}), p)), \quad \forall p \in M,
$$

where $\operatorname{conv}(\mathcal{F})$ denote the convex hull of $\mathcal{F}$.

In fact, the condition (5) says that $\mathcal{F}$ and its convex hull conv $(\mathcal{F})$ are equivalent by the definition in [12]. Now we are ready to prove the main theorem. Proof of Theorem $3^{2}$ We only need to prove local accessibility implies controllability. Let $\mathcal{E}=\mathcal{F} \cup\{-f\}$ and $\mathcal{L}(\mathcal{E})$ denotes the Lie algebra generated by $\mathcal{E}$. Then conv $(\mathcal{E})$ is symmetric and by the accessibility condition span $\mathcal{L}(\mathcal{E})(p)=T_{p}(M), \forall p \in M$. Also span $\mathcal{L}(\operatorname{conv}(\mathcal{E}))(p)=T_{p} M$. Since $\operatorname{conv}(\mathcal{E})$ is symmetric, by previous remark on symmetric systems, we have $\mathrm{R}(\operatorname{conv}(\mathcal{E}), p)=M, \forall p \in M$. From the above lemma, $\mathrm{dl}(\mathrm{R}(\mathcal{E}, p))=$ $M, \forall p \in M$, also. Since $\operatorname{span} \mathcal{L}(\mathcal{E})(p)=\operatorname{span} \mathcal{L}(-\mathcal{E})(p)=T_{p} M, \mathrm{R}(-\mathcal{E}, y)$ has a nonempty interior for all $y \in M$. Hence, for any $q \in M$, there exists $r \in \operatorname{int}(\mathbf{R}(-\mathcal{E}, q)) \cap \mathbf{R}(\mathcal{E}, p)$. This implies $q \in \mathbf{R}(\mathcal{E}, p)$. As a result, $\mathrm{R}(\mathcal{E}, p)=M, \forall p \in M$

Now, we prove that for arbitrary $p, q \in M, q \in \mathrm{R}(\mathcal{F}, p)$

Let $z \in$ int $\mathrm{R}(-\mathcal{F}, q)$ and $w \in$ int $\mathrm{R}(\mathcal{F}, p)$. Since $\mathrm{R}(\mathcal{E}, w)=M$, there exists an integral curve of $\mathcal{E}$ joining $w$ to $z$, i.e.,

$$
\begin{aligned}
\exists t_{1}, \ldots, t_{k} & >0 \text { and } X_{1}, \ldots, X_{k} \in \mathcal{E} \text { s.t. } \\
z & =\phi_{t_{1}}^{\mathrm{x}_{1}} \circ \cdots \circ \phi_{t_{k}}^{\mathrm{x}_{k}}(w) .
\end{aligned}
$$

If $X_{i}, i=1, \ldots, k$, belong to $\mathcal{F}$, we have $q \in \mathrm{R}(\mathcal{F}, p)$. If there are some $X_{i}$ such that $X_{i}=-f \in \mathcal{E}$, we now try to "correct" the integral curve by using the property of weakly positive. Poisson stability of $f$. Without loss of generality, assume that $X_{1}=-f$. We can find a neightborhood of $z$, say $U_{z}$, in the interior of $\mathrm{R}(-\mathcal{F}, q)$ such that

$$
\left(\phi_{t_{l}}^{\mathrm{x}_{1}} \circ \cdots \circ \phi_{t_{k}}^{\mathbf{x}_{k}}\right)^{-1}\left(U_{z}\right) \subset \operatorname{int}(R(\mathcal{F}, p)),
$$

with $w \in\left(\phi_{t_{1}}^{\mathrm{x}_{1}} \circ \cdots \circ \phi_{l_{k}}^{\mathrm{x}_{\mathrm{h}}}\right)^{-1}\left(U_{z}\right)$

Now the weakly positively Poisson stability of $f$ implies that for $U_{z}$ and $t_{1}$, there exists $T_{1}>t_{1}$ such that

$$
\phi_{T_{1}}^{f}\left(U_{2}\right) \cap U_{2} \neq \emptyset
$$

Accordingly, there exists $\xi$ and $\bar{\xi}$ such that $\xi, \bar{\xi} \in U_{z} \subset \operatorname{int}(\mathrm{R}(-\mathcal{F}, q))$ and $\xi=\phi_{T,}^{f}(\bar{\xi})$. From $(6)$, we can find $s \in \operatorname{int}(\mathrm{R}(\mathcal{F}, p))$, such that

$$
\bar{\xi}=\phi_{t_{1}}^{-f} \circ \cdots \circ \phi_{t_{k}}^{x_{k}}(s)
$$

As a result,

$$
\begin{aligned}
& \xi=\phi_{T_{1}}^{f} \circ \phi_{t_{1}}^{-f} \circ \cdots \circ \phi_{t_{k}}^{\mathbf{x}_{\mathbf{k}}}(s) \\
& =\phi_{T_{1}-t_{1}}^{J} \circ \cdots \circ \phi_{t_{k}}^{\mathrm{x}_{k}}(s)
\end{aligned}
$$

Since $\xi \in \operatorname{int}(\mathrm{R}(-\mathcal{F}, q))$ and $s \in \operatorname{int}(\mathrm{R}(\mathcal{F}, p))$, we proved

$$
q=\phi^{\mathcal{F}} \circ \phi_{T_{1}-t_{1}}^{f} \circ \cdots \circ \phi_{t_{k}}^{\mathrm{x}_{k}} \circ \phi^{\mathcal{F}}(s)
$$

where $\phi^{\mathcal{F}}$ denote a flow of $\mathcal{F}$. Other possible $-f$ in $X_{2}, \ldots, X_{k}$ can be treated in an analogous way. As a consequence, $q$ can be reached from $p$ by some

In $[4,19]$, it has been proved that the controllability can be established for Poisson stable systems satisfying the Lie algebra rank condition. The theorem above extends their results to the weakly positively Poisson stable theorem above extends their results to the weak y positively Poisson stable
systems. Now, we combine Theorem 1 and 3 to yield the following theorem.

Theorem 4 Consider a control system on $M$,

$$
\dot{x}=X_{H}+\sum_{i=1}^{j} g_{i} u_{i},
$$

where $X_{H}$ is an analytical Hamiltonian vector field with time-independent Hamiltonian $H$, and $g_{i}, i=1, \ldots, j$ are analytical vector fields on $M$. Assume $M$ is bounded, then the system ( () is controllable (with piecewise constant inputs) iff the Lie algebra rank condition is satisfied, i.e.,

$$
\operatorname{span} \mathcal{L}\left(X_{H}, g_{1}, \ldots, g_{j}\right)(p)=T_{p} M, \quad \forall p \in M .
$$

This theorem will be directly used in the following sections to prove the controllability of the spacecraft system by using gas jets.

${ }^{2}$ The following proof is mainly based on an unpublished note of W. P. Dayawansa.

\section{Controllability of Spacecraft System}

With the tools developed in the previous sections, we are now to prove the controllability result for the spacecraft system.

\subsection{Spacecraft System with Reaction Wheels}

First, we consider the system of spacecraft with reaction wheels. The equations of motion were given by (1). To apply Theorem 1 , it is natural to the dynamics on $T Q$ to the dynamics on $T^{*} Q$. We accomplish it through the following process. With conjugate momenta defined by

$$
\Pi=\mathbf{I} \mathbf{\Omega}+\eta, \quad \mathbf{h}=\eta+\sum_{i=1}^{l} I_{i} \mathbf{n}_{i} \mathbf{n}_{i}^{\top} \Omega, \quad \mathbf{p}=m \mathbf{r},
$$

where $\eta$ is defined in (2), we can transform the dynamical equations (1) to

$$
\begin{aligned}
& \dot{B}=B\left(\mathbf{J}^{-1}(\Pi-\mathbf{h})\right) \\
& \dot{\Pi}=\Pi \times \mathbf{J}^{-1}(\Pi-\mathbf{h})+\int_{B} \frac{\mu\left(B^{\mathrm{T}} \mathbf{r} \times \bar{q}\right)}{|\mathbf{r}+B \tilde{q}|^{3}} d m(\ddot{q}), \\
& \dot{\mathbf{r}}=\frac{\mathbf{p}}{m} \\
& \dot{\mathbf{p}}=-\int_{B} \frac{\mu(\mathbf{r}+B \bar{q})}{|\mathbf{r}+B \bar{q}|^{3}} d m(\bar{q}), \\
& \dot{\mathbf{h}}=\tau,
\end{aligned}
$$

where $\tau=\sum_{i=1}^{\prime} \tau_{i} \mathbf{n}_{i}$. Here, $\Pi$ denotes the total angular momentum of the assembly and $\mathbf{J} \triangleq \mathrm{I}-\sum_{i=1}^{l} I_{i} \mathbf{u}_{i} \mathbf{u}_{i}^{\top}$

For the case of no control inputs, i.e., $\tau_{i}=0$ (sometimes this is called the case of free rotors), then $\mathbf{h}$ is constant. Hence, the dynamical system described above is indeed a canonical Hamiltonian system with associated described above

$$
H(B, B \hat{\Pi}, \mathbf{r}, \mathbf{p})=\frac{1}{2}<\Pi-\mathbf{h}, \mathbf{J}^{-1}(\Pi-\mathbf{h})>+\frac{1}{2} \frac{\mathbf{p}^{2}}{m}-\int_{\mathcal{B}} \frac{\mu}{|\mathbf{r}+B \bar{q}|} d m(\bar{q}) .(9)
$$

Accordingly, the following proposition is obtained:

Proposition 1 The dynamics of the spacecraft system with free rotors can be expressed as a canonical Hamilton's equation in $(B, B \hat{\Pi}, \mathbf{r}, \mathbf{p})$-coordinates with Hamiltonian $(9)$, where $\mathbf{h}$ is constant. Moreover, when $\mathbf{r}$ is finite and bounded away from zero, then the system is weakly positively Poisson stable.

Proof The restriction on $\mathbf{r}$ implies that the energy manifold is bounded, and then the system is weakly positively Poisson stable by theorem 1 .

For the controllability of system ( 8 ) being of concern, we look at the correspondiug conservation law of angular momentum. In fact, it can be easily verified that

$$
B \Pi+\mathbf{r} \times \mathbf{p}=\mathbf{k}
$$

is the total angular momentum with respect to the inertial frame and is conserved for (8). Accordingly, the relationship among $\Pi, r, P$ is invariant for any control input $\tau$. We thus conclude that the control system $(8)$ is not controllable.

However, the phase variables that we are concerned are not $(B, B \hat{\Pi}, \mathbf{r}, \mathbf{p})^{\top}$ where II is the total angular momentum of the spacecraft and reaction wheels, but instead $\left(B, B \hat{\Pi}_{s}, \mathbf{r}, \mathbf{p}\right)^{\top}$, where $\Pi,=\mathbf{J} \Omega$ denotes the angular momentum of the spacecraft with locked reaction wheels. The dynamical equations (1) can be transformed into a system in terms of the variables $\left(B, B \hat{\Pi}_{s}, \mathbf{r}, \mathbf{p}\right)$ as follows.

$$
\begin{aligned}
\dot{B} & =B \widehat{\mathbf{J}}^{-1} \Pi_{s}, \\
\dot{\Pi}_{s} & =\left(\mathrm{\Pi}_{s}+\mathbf{l}\right) \times \mathbf{J}^{-1} \Pi_{s}+\int_{\mathcal{B}} \frac{\mu\left(B^{\top} \mathbf{r} \times \tilde{q}\right)}{|\mathbf{r}+B \bar{q}|^{3}} d m(\bar{q})-\tau \\
\dot{\mathbf{r}} & =\frac{\mathbf{p}}{m} \\
\dot{\mathbf{p}} & =-\int_{B} \frac{\mu(\mathbf{r}+B \bar{q})}{|\mathbf{r}+B \bar{q}|^{3}} \ln (\bar{q}), \\
\dot{\mathbf{h}} & =\tau .
\end{aligned}
$$

In contrast with the system ( 8 ), the constant of motion for system (11) be $B\left(\Pi_{s}+\mathbf{h}\right)+\mathbf{r} \times \mathbf{p}=\mathbf{k}$,

where the relationship among $B, \Pi_{b}, r_{1}, p$ is no longer constrained since $\mathbf{h}_{\mathbf{h}}$ can be controlled through $\tau$. It is noteworthy that there is no obvious Hamiltonian structure for the spacecraft system (11) with zero input. Thus Theorem 1 can not be directly applied to system (11). While the system (8) is not 1 can not be directly applied to system (11). While the system (8) is not controllable, its property of weakly positive Poisson stability can be used to
help us in establishing such property for (11). This is achieved through the help us in establishing such property 
Definition $3[2,1]$ Topological flows $\phi$ and $\psi$ on phase space $M, N$, respectively, are said to be topologically equivalent if there is a homeomorphism $\Phi: N \rightarrow M$ which carries the trajectories of one flow to the trajectories of the other while preserving the direction of motion.

Proposition 2 Consider vector fields $X, Y$ and associated flow $\phi^{x}$ nad $\phi^{y}$, respectively. If $\phi^{x}$ and $\phi^{y}$ are topologically equivalent, then the weakly positive Poisson stability of $X$ implies the weakly positive Poisson stability of $Y$, and
vice versa.

Proof By the topological equivalence, there is a homeomorphism $\Phi$ with $M=\Phi(N)$ such that for any $y \in N$ and every neighborhood $V_{y} \subset N$, the set $U_{x}=\Phi\left(V_{y}\right)$ is a neighborhood of $x=\Phi(y)$. Since $X$ is weakly positively Poisson stable, for some $T<\infty, \phi_{T}^{\mathrm{x}}\left(U_{x}\right) \cap U_{x} \neq \emptyset$. From the property that $\Phi$ carries the trajectories of $Y$ to the trajectories of $X$ while preserves the $\Phi$ carries the trajectories of $Y$ to the trajectories of $X$ while preserves the
direction of motion, we know $\phi_{1}^{y}\left(V_{y}\right) \cap V_{y} \neq \emptyset$ for some $t \leq T$. Corollary 1 The drift vector field of system (11) is weakly positively Poisson stable.

Proof: The corollary follows if we can establish the topological equivalence between the flows of the drift dynamics for (8) and (11). Let

$$
\Phi:\left(\begin{array}{c}
B \\
\Pi, \\
\mathbf{r} \\
\mathbf{p}
\end{array}\right) \mapsto\left(\begin{array}{c}
B \\
\Pi \\
\mathbf{r} \\
\mathbf{p}
\end{array}\right)=\left(\begin{array}{c}
B \\
\Pi_{s}+\mathbf{h} \\
\mathbf{r} \\
\mathbf{p}
\end{array}\right),
$$

where $\mathbf{h}$ is a constant. The mapping $\Phi$ is obviously homeomorphism. Now we prove that the transformation $\Phi$ carries the trajectories. Let $\phi_{t}^{x}$ and $\phi_{t}^{y}$ denote the flows of the drift dynamics $(8)$ and $(11)$, respectively. For arbitrary $y=\left(B, \Pi_{s}, \mathbf{r}, \mathbf{p}\right)^{\top}$, we need to verify $\left.\frac{d}{d i}\left(\Phi \circ \phi_{t}^{y}\right)\right|_{y}=X(\Phi(y))$. In particular,

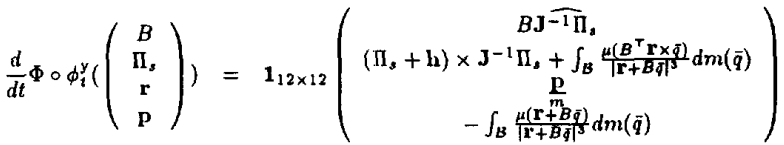

$$
\begin{aligned}
& =X\left(\left(\begin{array}{c}
B \\
\mathrm{n} \mathbf{s}+\mathbf{h} \\
\mathbf{r} \\
\mathbf{p}
\end{array}\right)\right)
\end{aligned}
$$

Accordingly, $\phi^{\mathrm{x}}$ and $\phi^{\mathrm{y}}$ are topologically equivalent, and then the corollary follows from Proposition 2

As stated before, we are concerned with the controllability property on the space $\left(B, B \hat{\Pi}_{s}, \mathbf{r}, \mathbf{p}\right)^{\top}$. The dynamics of $\mathbf{h}$ in (11) can be in fact decoupled from the dynamics of $\left(B, B \hat{\Pi}_{s}, \mathbf{r}, \mathbf{p}\right)$ by using the law of conservation of angular momentum (12). Accordingly, for constant $\mathbf{k}$, we reformulate the system as follows

$$
\begin{aligned}
\dot{B}= & B \mathbf{J}^{-1} \Pi_{s}, \\
B \hat{\Pi}_{s}= & B \mathbf{J}^{-1} \Pi_{s} \hat{\Pi}_{s}+ \\
& B\left(B^{\top}(\mathbf{k}-\mathbf{r} \times \mathbf{p}) \times \mathbf{J}^{-1} \Pi_{s}+\int_{B} \frac{\mu\left(B^{\top} \mathbf{r} \times \bar{q}\right)}{|\mathbf{r}+B \bar{q}|^{3}} d m(\bar{q})\right)-B \hat{\tau} \\
\dot{\mathbf{r}}= & \frac{\mathbf{p}}{n}, \\
\dot{\mathbf{p}}= & -\int_{B} \frac{\mu(\mathbf{r}+B \bar{q})}{|\mathbf{r}+B \bar{q}|^{3}} d m(\bar{q}),
\end{aligned}
$$

where the canonical representation on $T T^{*}\left(S O(3) \times \mathbb{R}^{3}\right)$ (cf. [18]) has been adopted. The drift dynamics of (13) is also weakly positively Poisson stable. This follows from the corresponding property of the drift dynamics of (11) In fact, for arbitrary constant $\mathbf{k}$ in (13), we can find $\mathbf{h}=B^{\top}(\mathbf{k}-\mathbf{r} \times \mathbf{p})-\Pi$, which is also a constant such that the drift dynamics of (13) is the same as that of (11).

Now, we prove the controllability of dynamics (13) by applying Theorem 3. The Lie algebra rank condition for (13) can be checked as follows. Since the Lie algebra rank condition is invariant under control feedback, we can choose

$$
\tau=-B^{\top}(\mathbf{k}-\mathbf{r} \times \mathbf{p}) \times \mathbf{J}^{-1} \Pi_{s}-\int_{\mathbf{B}} \frac{\mu\left(B^{\top} \mathbf{r} \times \bar{q}\right)}{|\mathbf{r}+B \bar{q}|^{3}} d m(\bar{q})-\mathbf{u},
$$

Here, we assume that $\tau$ can control three independent directions of rotation With the feedback (14), system (13) can be put into the following form:

$$
\begin{aligned}
\dot{B} & =B \widehat{J}^{-1} \Pi_{s}, \\
B \dot{\mathbf{\Pi}}, & =B \hat{J}^{-1} \hat{\Pi}_{s},+B \hat{\mathbf{H}}_{,} \\
\dot{\mathbf{r}} & =\frac{\mathbf{p}}{m}, \\
\dot{\mathbf{p}} & =-\int_{\mathcal{B}} \frac{\mu(\mathbf{r}+B \bar{q})}{|\mathbf{r}+B \bar{q}|^{3}} d m(\bar{q}) .
\end{aligned}
$$

Furthermore, by writing $\mathbf{u}=\mathbf{b}_{1} u_{1}+\mathbf{b}_{2} u_{2}+\mathbf{b}_{3} u_{3}$, we could express the above equation in the form of (4) with

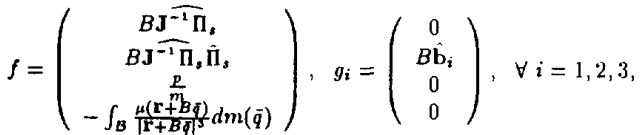

where $\mathbf{b}_{1}=(1,0,0)^{\top}, \mathbf{b}_{2}=(0,1,0)^{\top}, \mathbf{b}_{3}=(0,0,1)^{\top}$. By applying the techniques developed in $[17,18]$, the Lie bracket of $f$ and $g_{i}$ results

$$
f_{i} \triangleq\left[f, g_{i}\right]=\left(\begin{array}{c}
-B \widehat{J}^{-1} \mathbf{b}_{i} \\
-B \mathbf{J}^{-1} \mathbf{b}_{i} \hat{\Pi}_{s} \\
0 \\
0
\end{array}\right), \text { for } i=1,2,3
$$

Similarly, we have the following vector fields generated by the Lie bracket operation, $\forall i=1,2,3$,

$$
\begin{aligned}
& F_{i} \triangleq\left[f, f_{i}\right]=\left(\begin{array}{c}
-B\left(\mathbf{J}^{-1} \Pi_{s} \times \mathbf{J}^{-1} \mathbf{b}_{i}\right)^{-} \\
-B\left(\mathbf{J}^{-1} \Pi_{s} \times \mathbf{J}^{-1} \mathbf{b}_{i}\right)^{-\hat{\Pi}_{s}} \\
0 \\
\mu \int_{\mathcal{B}}\left(\frac{1}{\left|\mathbf{r}+B_{q}\right|^{s}}-\frac{3\left(\mathbf{r}+B_{g} \mathbf{r}^{\top}\right.}{|\mathbf{r}+B g|^{3}}\right) B \hat{\bar{q}} d m(\tilde{q}) \mathbf{J}^{-1} \mathbf{b}_{i}
\end{array}\right), \\
& G_{i} \triangleq\left[f, F_{i}\right]=\left(\begin{array}{c}
* \\
* \\
-\frac{F_{i, 4}}{m} \\
*
\end{array}\right),
\end{aligned}
$$

where "*" denotes the "don't care" terms and $F_{i, 4}$ is the fourth component of the vector field $F_{i}$ given by

$$
\mu \int_{\mathcal{B}}\left(\frac{1}{|\mathbf{r}+B \bar{q}|^{3}}-\frac{3(\mathbf{r}+B \bar{q}) \mathbf{r}^{\top}}{|\mathbf{r}+B \bar{q}|^{5}}\right) B \hat{\bar{q}} d m(\bar{q}) \mathrm{J}^{-1} \mathbf{b}_{i} .
$$

From the above formulae, it is noted that the Lie algebra rank condition is satisfied if the three vectors $F_{1,4}, F_{2,4}, F_{3,4}$ can span the 3-dimensional space. To this end, we rewrite $\sum_{i=1}^{3} c_{i} F_{i, 4}$ witl $c_{i} \in \mathbb{R}$ as follows,

$\mu B \underbrace{\int_{\mathcal{U}}\left(\frac{\hat{\bar{q}}}{|\lambda+\bar{q}|^{3}}-\frac{3 \lambda \lambda^{\top} \hat{\bar{q}}}{|\lambda+\bar{q}|^{3}}+\frac{3 \bar{q} \bar{q}^{\top} \hat{\lambda}}{|\lambda+\bar{q}|^{5}}\right) d m(\bar{q})}_{P} \sum_{i=1}^{3} c_{i} \mathbf{J}^{-1} \mathbf{b}_{\mathbf{i}} \triangleq \mu B P \sum_{i=1}^{3} c_{i} \mathbf{J}^{-1} \mathbf{b}_{i}$

where $\lambda=B^{\top} \mathbf{r}$. Now, it becomes obvious that the non-singularity of matrix $P$ at every $(B, \mathbf{r})$ implies the Lie algebra rank condition which, in turn, implies the controllability. However, for an asymmetric molecule, which consists of six point masses with two on each principal axis (c.f. [31]), matrix $P$ can be computed to be singular for some configurations. Therefore, a more detailed analysis is necessary.

Let $\mathbf{J}^{-1} \mathbf{b}_{\mathbf{i}} \triangleq \overline{\mathbf{b}}_{\mathbf{i}}$ and $\boldsymbol{I}$ denote the coefficient of inertial of the assembly, i.e., $I \triangleq \int_{B} \bar{q} \bar{q}^{\top} d m(\bar{q})$. We have $I=\frac{1}{2} \operatorname{tr}(I) 1-I$ where $I$ is the total moment of inertia of the assembly and is assumed to be diagonal. Hence $\mathcal{I}$ is a diagonal matrix and has three different eigenvalues, say $\mathcal{I}_{1}, \mathcal{I}_{2}, \mathcal{I}_{3}$. For most applications, the body size of the spacecraft is very small comparing to the size of the orbital radius. We could thus approximate our vector fields based on the small parameter $\epsilon \triangleq \frac{\max |q|}{|\lambda|}$ which represent the ratio of body size to the radius. Taking Taylor expansion with respect to $\epsilon$, the matrix $P$ can be
expressed as

$$
\begin{aligned}
& P=\underbrace{\frac{-3 \widehat{\mathcal{I}} \lambda}{|\lambda|^{5}}}_{O\left(c^{2}\right)}+\underbrace{\int_{B} \frac{-3 \hat{\bar{q}}\left(\bar{q}^{\top} \bar{q}\right)}{2|\lambda|^{5}} d m(\bar{q})+\int_{B} \frac{15 \hat{\bar{q}}\left(\lambda^{\top} \bar{q}\right)^{2}}{2|\lambda|^{7}} d m(\bar{q})}_{O\left(x^{3}\right)}+ \\
& \underbrace{\frac{15 \lambda \lambda^{\top} \widehat{\mathcal{I} \lambda}}{|\lambda|^{\top}}}_{\mathcal{O}\left(\varepsilon^{2}\right)}+\underbrace{\int_{\mathcal{B}} \frac{15 \lambda \lambda^{\top} \hat{\bar{q}}\left(\bar{q}^{\top} \bar{q}\right)}{2|\lambda|^{\top}} d m(\bar{q})+\int_{\mathcal{B}} \frac{-105 \lambda \lambda^{\top} \hat{\bar{q}}\left(\lambda^{\top} \bar{q}\right)^{2}}{2|\lambda|^{9}} d m(\bar{q})}_{\mathcal{O}\left(\varepsilon^{3}\right)}+ \\
& \underbrace{\frac{3 \tau \hat{\lambda}}{|\lambda|^{5}}}_{\mathcal{O}\left(\epsilon^{2}\right)}+\underbrace{\int_{\mathcal{B}} \frac{-15 \bar{q} \bar{q}^{\top} \hat{\lambda}\left(\lambda^{\top} \bar{q}\right)}{|\lambda|^{7}} d m(\bar{q})}_{\mathcal{O}\left(\epsilon^{3}\right)}+\mathcal{O}\left(\epsilon^{4}\right) .
\end{aligned}
$$

For the second-order approximations, it can be found that the determinant

$$
\operatorname{det} P=\frac{-9}{|\lambda|^{5}}\left(\mathcal{I}_{1}-\mathcal{I}_{2}\right)\left(\mathcal{I}_{2}-\mathcal{I}_{3}\right)\left(\mathcal{I}_{3}-I_{1}\right) \lambda_{1} \lambda_{2} \lambda_{3},
$$

with $\lambda=\left(\lambda_{1}, \lambda_{2}, \lambda_{3}\right)^{\top}$. Accordingly, for nonzero $\lambda_{1}, \lambda_{2}, \lambda_{3}$, and asymmetric body, the Lie algebra rank condition is satisfied. However, in order to apply Theorem 3 , Lie algebra rank condition needs to be satisfied for all $\lambda_{1}, \lambda_{2}, \lambda_{3}$. 
Theorem 5 Suppose the spacecraft system (19) with three-axes reaction wheel $s$, i.e., the effects of reaction wheels are on three independent axes. Suppose also that $\mathrm{r}$ is finite and sufficiently large comparing to the size of the spacecraft. Then the spacecrafi system is controllable if the spacecrafi has three
distinct principal moments of inertia and its mass distribution satisfies the constraints

$$
\int_{B} \bar{q}^{\top}\left(-1+5 \mathbf{b}_{i} \mathbf{b}_{i}^{\top}\right) \bar{q} \bar{q}^{\top} d m(\bar{q}) \quad \forall \quad \mathbf{b}_{i}, \forall i=1,2,3 .
$$

Moreover, if the total effects of reaction wheels are only on one-axis or two-independent axis, then the spacecrafi system is neither controllable nor accessible.

Proof The weakly positive Poisson stability of the drift dynamics of (13) has been proved. For the Lie algebra rank condition, it is easily checked that $g_{i}, f_{i}, \forall i=1,2,3$, span $T_{(B, B \Pi)} T^{*} S O(3)$. It suffices to seek some vector fields which span $T_{(\mathbf{r}, \mathbf{p})} T^{*} \mathbb{R}^{3}$.

The matrix $P$ in (18) can be expressed as component form of

$\underbrace{\frac{3}{|\lambda|^{5}}\left(\begin{array}{ccc}\frac{5 \lambda_{1} \lambda_{2} \lambda_{3}}{|\lambda|^{2}}\left(I_{3}-I_{2}\right) & \left(\frac{5 \lambda_{1}^{2} \lambda_{3}}{|\lambda|^{2}}-\lambda_{3}\right)\left(I_{1}-I_{3}\right) & \left(\frac{5 \lambda_{1}^{2} \lambda_{2}}{|\lambda|^{2}}-\lambda_{2}\right)\left(I_{2}-I_{1}\right) \\ \left(\frac{3 \lambda_{2}^{2} \lambda_{3}}{|\lambda|^{2}}-\lambda_{3}\right)\left(I_{3}-I_{2}\right) & \frac{5 \lambda_{1} \lambda_{3} \lambda_{3}}{|\lambda|^{2}}\left(I_{1}-I_{3}\right) & \left(\frac{5 \lambda_{3}^{2} \lambda_{1}}{|\lambda|^{2}}-\lambda_{1}\right)\left(I_{2}-I_{1}\right) \\ \left(\frac{5 \lambda_{3}^{2} \lambda_{2}}{|\lambda|^{2}}-\lambda_{2}\right)\left(I_{3}-I_{2}\right) & \left(\frac{3 \lambda_{3}^{3} \lambda_{2}}{|\lambda|^{2}}-\lambda_{1}\right)\left(I_{1}-I_{3}\right) & \frac{5 \lambda_{1} \lambda_{2} \lambda_{3}}{|\lambda|^{2}}\left(I_{2}-I_{1}\right)\end{array}\right)}_{\mathcal{O}\left(c^{2}\right)}$

$$
+\underbrace{\left(\begin{array}{ccc}
\epsilon_{11}^{\prime} & \epsilon_{12}^{\prime} & \epsilon_{13}^{\prime} \\
c_{21}^{\prime} & \epsilon_{22}^{\prime} & \epsilon_{23}^{\prime} \\
\epsilon_{31}^{\prime} & \epsilon_{32}^{\prime} & \epsilon_{33}^{\prime}
\end{array}\right)}_{\mathcal{O}\left(\epsilon^{3}\right)}
$$

The proof of the theorem is conducted through the following four steps. The idea is to find three independent vectors on the tangent space corresponding to $\mathbf{r}$ and $p$, respectively.

Step 1: We show that for every configuration $\left(B, B \hat{\Pi}_{s}, \mathbf{r}, \mathbf{p}\right)^{\top}$ there exists at least one vector $\nu \in \mathbb{R}^{3}$ satisfying $\nu \bigvee \lambda \lambda$ and $\nu, \lambda$.

- Case 1: $\left|\lambda_{1}\right|,\left|\lambda_{2}\right|,\left|\lambda_{3}\right|>\tilde{\epsilon}$, where $\tilde{\epsilon}$ is a small number. This condition in fact says that $\lambda_{1} \lambda_{3}$ are all reasonably large. Under this cirin fact says that $\lambda_{1}, \lambda_{2}, \lambda_{3}$ are all reasonably large. Under this cir
cumstance, the matrix $P$ is nonsingular. Hence, by suitably assigning $c_{1}, c_{2}, c_{3}$, we can find $\nu=P \sum_{i=1}^{3} c_{i} \bar{b}_{i}$ such that $\nu \forall \lambda$ and $\nu A \lambda$.

- Case 2: Without loss of generality, assume $\lambda_{1} \in(-\tilde{\epsilon}, \tilde{\epsilon}),\left|\lambda_{2}\right|,\left|\lambda_{3}\right|>\tilde{\epsilon}$, or $\lambda_{1}$ small and $\lambda_{2}, \lambda_{3}$ reasonably large. Let $\sum_{i=1}^{3} c_{i} \breve{b}_{i}=\left[c_{1}^{\prime}, c_{2}^{\prime}, 0\right]^{\top}$ Obviously, $c_{1}^{\prime}, c_{2}^{\prime}$ can be chosen arbitrarily. We compute

Hence we have

$$
\nu=P\left(\begin{array}{c}
c_{1}^{\prime} \\
c_{2}^{\prime} \\
0
\end{array}\right) \approx \frac{3}{|\lambda|^{5}}\left(\begin{array}{c}
-c_{2}^{\prime}\left(\mathcal{I}_{1}-\mathcal{I}_{3}\right) \lambda_{3} \\
c_{1}^{\prime}\left(\mathcal{I}_{3}-\mathcal{I}_{2}\right)\left(\frac{5 \lambda_{2}^{2} \lambda_{3}}{1 \lambda^{2}}-\lambda_{3}\right) \\
c_{1}^{\prime}\left(\mathcal{I}_{3}-\mathcal{I}_{2}\right)\left(\frac{5 \lambda_{3}^{2} \lambda_{2}}{\left.T \lambda\right|^{2}}-\lambda_{2}\right)
\end{array}\right)
$$

$$
\begin{aligned}
& \nu \cdot \lambda \approx \frac{12 c_{1}^{\prime}\left(\mathcal{I}_{3}-\mathcal{I}_{2}\right)}{|\lambda|^{5}} \lambda_{2} \lambda_{3}, \\
& \nu \times \lambda \approx\left(\begin{array}{c}
c_{1}^{\prime}\left(\mathcal{I}_{3}-\mathcal{I}_{2}\right)\left(\lambda_{2}^{2}-\lambda_{3}^{2}\right) \\
-c_{2}^{\prime}\left(\mathcal{I}_{1}-I_{3}\right) \lambda_{3}^{2} \\
-c_{2}^{\prime}\left(I_{1}-I_{3}\right) \lambda_{2} \lambda_{3}
\end{array}\right) .
\end{aligned}
$$

Accordingly, it is evident that we can choose $c_{1}^{\prime}, c_{2}^{\prime}$ such that $\nu \not \lambda$ and $\nu \perp \lambda$.

- Case 3: $\lambda_{1}, \lambda_{2} \in(-\tilde{\epsilon}, \tilde{\epsilon}), \lambda_{3} \approx|\lambda|$. Assume either $\lambda_{1}$ or $\lambda_{2}$ is nonzero, say $\lambda_{2} \neq 0$. By a similar argument as the above case, we can choose $\sum^{3} \lambda_{i} \bar{b}_{i}=\left[c_{1}^{\prime}, 0,0\right]^{\top}$ such that $\nu=P \sum^{3} c_{i} \overline{\mathbf{b}}_{i}$ satisfies $\nu y \lambda$ and $\sum_{i=1}^{3} c_{i} \bar{b}_{i}=\left[c_{1}^{\prime}, 0,0\right]^{\top}$ such that $\nu=P \sum_{i=1}^{3} c_{i} \bar{b}_{i}$ satisfies $\nu, y \lambda$ and
$\nu, \lambda$. When $\lambda_{1}=\lambda_{2}=0$, the above arguments do not work. We need to look at the third-order approximation, i.e., the terms of $\mathcal{O}\left(\epsilon^{3}\right)$ in $P$ need to be used. We have

$$
\begin{aligned}
& \lambda^{\top} P \\
= & \frac{-9}{|\lambda|^{5}} \lambda^{\top} \mathcal{I} \hat{\lambda}+\frac{1}{|\lambda|^{5}} \int_{\mathcal{B}}\left(-6 \bar{q}^{\top} \bar{q} \bar{q}^{\top} \hat{\lambda}+\frac{30\left(\lambda^{\top} \bar{q}\right)^{2} \bar{q}^{\top} \hat{\lambda}}{|\lambda|^{2}}\right) d m(\bar{q})+\mathcal{O}\left(\epsilon^{4}\right), \\
\approx & \left\{\frac{6}{|\lambda|^{5}} \int_{\mathcal{B}} \bar{q}^{\top}\left(-1+\frac{5 \lambda \lambda^{\top}}{|\lambda|^{2}}\right) \bar{q} \bar{q}^{\top} d m(\bar{q})\right\} \hat{\lambda},
\end{aligned}
$$

where the fact $\lambda \| I \lambda$ has been used. Now, let $\nu_{1}=P \sum_{i=1}^{3} c_{i} \bar{b}_{i}$, we have

$$
\lambda^{\top} \nu_{1} \approx\left\{\frac{6}{|\lambda|^{5}} \int_{\mathcal{B}} \bar{q}^{\top}\left(-1+5 \mathbf{b}_{3} \mathbf{b}_{3}^{\top}\right) \bar{q} \bar{q}^{\top} d m(\tilde{q})\right\} \hat{b}_{3} \sum_{i=1}^{3} c_{i} \overline{\mathbf{b}}_{i}
$$

Thus, if conditions (19) are satisfied, we can choose $c_{i}, i=1,2,3$, such that $\nu_{1} / \perp \lambda$. Now, if $\nu_{1} \lambda \mid \lambda$, we are done and the desired $\nu=\nu_{1}$. Otherwise, by similar argument as in Case 2 above, we can find $\nu_{2}$ such that $\nu_{2} \not \lambda$. If $\nu_{2} \not \lambda$, we let $\nu=\nu_{2}$. If $\nu_{2} \perp \lambda$, then we pick $\nu=\nu_{1}+\nu_{2}$ Obviously, $\nu h \lambda$ and $\nu \perp \lambda$. As a result, $\nu$ is the desired vector.

In summary $y_{1}$ we make the following conclusion: let $\Delta_{1}$ denote the distribution spanned by vector fields $F_{i}, i=1,2,3$ in (16) then there exists a vector field $\mathbf{V}_{1}, \mathbf{V}_{1} \neq 0$, and $\mathbf{V}_{1,4}\left(B, B \hat{\Pi}_{s}, \mathbf{r}, \mathbf{p}\right)=B \nu$ with $\nu \not \lambda$ and $\nu A \lambda$, such that

$$
\Delta_{1} \supset \operatorname{span}\left\{\left(*, *, 0, V_{1}\right)^{\top}\right\}
$$

for every point of $\left(B, \hat{\Pi}_{s}, \mathbf{r}, \mathbf{p}\right)$.

Step 2: With $\nu$ found in Step 1, here we prove that $\lambda \times \nu$ is in $\operatorname{span}\left(\mathcal{L}\left(f, g_{i}\right)\right)$. Taking the Lie bracket of $F_{i}$ and $f_{i}$, we obtain

$$
\Delta_{2} \triangleq \operatorname{span}\left\{\left[\sum_{i=1}^{3} c_{i} F_{i}, \sum_{j=1}^{3} l_{j} f_{j}\right]\right\}=
$$

$\operatorname{span}\left\{\sum_{i=1}^{3} \sum_{j=1}^{3}\left(\begin{array}{c}* \\ 0 \\ -\mu B\left(-\widehat{l}_{j} \overline{\mathbf{b}}_{j}\right) P(B, \mathbf{r}) c_{i} \overline{\mathbf{b}}_{i}+\mu B \frac{d}{d t_{i=0}} P\left(B e^{-t l_{j} \overline{\mathbf{b}}_{j}}, \mathbf{r}\right) c_{i} \overline{\mathbf{b}}_{i}\end{array}\right)\right\}$

For the scalars $c_{i}, i=1,2,3$ corresponding to the vector $\nu$ obtained in the previous step, we have

$$
\left[\sum_{j=1}^{3} c_{i} F_{i}, \sum_{j=1}^{3} l_{j} f_{j}\right]_{4}=\mu B \sum_{j=1}^{3} \widehat{l_{j}} \widehat{\mathbf{b}}_{j} \nu-\mu B\left(D_{\lambda} P \cdot \sum_{j=1}^{3} \widehat{l_{j} \widehat{\mathbf{b}}_{j} \lambda}\right) \nu,
$$

where $D_{\lambda} P$ denotes the derivative of $P$ with respect to $\lambda$. In fact, as shown in (17), $P$ is function of $\lambda=B^{\top} \mathbf{r}$ only. Now, we pick $l_{j}$ such that $\sum_{j=1}^{3} l_{j} \overline{\mathbf{b}}_{j}=$ $\kappa_{1} \lambda$. Then

$$
\left[\sum_{i=1}^{3} c_{i} F_{i}, \sum_{j=1}^{3} l_{j} f_{j}\right]=\mu B \kappa_{1}(\lambda \times \nu)
$$

As a result, if we define a vector field $\mathbf{V}_{2}$ such that $\mathbf{V}_{2,4}\left(B, B \hat{\Pi}_{s}, \mathbf{r}, \mathbf{p}\right)=$ $B(\lambda \times \nu)$, then we have

$$
\Delta_{1}+\Delta_{2} \supset \operatorname{span}\left\{\left(*, *, 0, \mathbf{V}_{1}\right)^{\top},\left(*, *, 0, \mathbf{V}_{2}\right)^{\top}\right\}
$$

Step 3: Now we proceed to construct the third independent vector field. By similar process as in the above step, we compute the Lie bracket

$$
\Delta_{3} \triangleq \operatorname{span}\left\{\left[\left[\sum_{i=1}^{3} c_{i} F_{i}, \sum_{j=1}^{3} l_{j} f_{j}\right], \sum_{k=1}^{3} d_{k} f_{k}\right]\right\}
$$

where $c_{i}, l_{j}, i, j=1,2,3$ were given in Step 1 and 2 , respectively. By choosing $d_{k}, k=1,2,3$ such that $\sum_{k=1}^{3} d_{k} \overline{\mathbf{b}}_{k}=\kappa_{2} \lambda$, the bracket can be found to be

$$
\left[\left[\sum_{i=1}^{3} c_{i} F_{i}, \sum_{j=1}^{3} l_{j} f_{j}\right], \sum_{k=1}^{3} d_{k} f_{k}\right]_{4}=\mu^{2} \kappa_{1} \kappa_{2} B(\lambda \times(\lambda \times \nu)) .
$$

Now, let a vector fiejd $\mathbf{V}_{3}$ be such that $\mathbf{V}_{3,4}=B(\lambda \times(\lambda \times \nu))$. Since $\{\nu, \lambda \times \nu, \lambda \times(\lambda \times \nu)\}$ forms a basis in $\mathbb{R}^{3}$, we conclude that the distribution $\Delta_{1}+\Delta_{2}+\Delta_{3}$ span the tangent space corresponding to the component p. Step 4: Now, we need to prove that the distribution $\operatorname{span}\left(\mathcal{L}\left(f, g_{i}\right)\right)$ span the tangent space corresponding to the component $\mathbf{r}$. By a similar argument as in the previous steps, we can show that the distribution

$$
\operatorname{span}\left\{\sum_{i=1}^{3} c_{i} G_{i},\left[\sum_{i=1}^{3} c_{i} G_{i}, \sum_{i=j}^{3} l_{j} f_{j}\right],\left[\left[\sum_{i=1}^{3} c_{i} G_{i}, \sum_{j=1}^{3} l_{j} f_{j}\right], \sum_{k=1}^{3} d_{k} f_{k}\right]\right\}
$$

where $c_{i}, l_{j}, d_{k}$ are chosen in Step $1-3$, spans the tangent space corresponding to $r$. Consequently, we have the following conclusion:

$$
\begin{aligned}
& \operatorname{span}\left\{g_{1}, g_{2}, g_{3}, f_{1}, f_{2}, f_{3},\right. \\
& \sum_{i=1}^{3} c_{i} F_{i},\left[\sum_{i=1}^{3} c_{i} F_{i}, \sum_{j=1}^{3} l_{j} f_{j}\right],\left[\left[\sum_{i=1}^{3} c_{i} F_{i}, \sum_{j=1}^{3} l_{j} f_{j}\right], \sum_{k=1}^{3} d_{k} f_{k}\right], \\
& \left.\sum_{i=1}^{3} c_{i} G_{i},\left[\sum_{i=1}^{3} c_{i} G_{i}, \sum_{j=1}^{3} l_{j} f_{j}\right],\left[\left[\sum_{i=1}^{3} c_{i} G_{i}, \sum_{j=1}^{3} l_{j} f_{j}\right], \sum_{k=1}^{3} d_{k} f_{k}\right]\right\} \\
& =T_{(B, B \hat{\Pi}, \mathbf{r}, \mathbf{p})} T^{T^{*}} S E(3),
\end{aligned}
$$


This complete the proof for the controllability of the system with three-axis reaction wheels.

On the other hand, for the case of one-axis or two-axis reaction wheels, there exists some vector $c$ such that

$$
c^{\top} B^{\top}(\mathbf{k}-\mathbf{r} \times \mathbf{p})-c^{\top} \Pi_{s}=c^{\top} \mathbf{h}=0 .
$$

Therefore, there exists some constant of motion $c^{\top} B^{\top}(\mathbf{k}-\mathbf{r} \times \mathbf{p})-c^{\top} \boldsymbol{I}_{s}$ which is invariant under any control input. Accordingly, the system (11) which is invariant under any control input. Accordingly, the system (11) with one-axis or two-axis reaction wheels cannot be accessible, and hence
not controllable. not controllable.

The conditions stated in the above theorem mainly require that the shape of the spacecraft should be not symmetric. In fact, for a totally symmetric spacecraft, the orbital dynamics is decoupled from attitude dynamics and is thus uncontrollable from the torque affecting the attitude motion. By choosing suitable masses and sizes of the molecule mentioned before, it has been checked that the conditions (19) can be satisfied. This shows that there are indeed some physical cases that the above theorem can be applied. Now we turn our attention to the controllability problem for the spacecraft system with gas jets.

\subsection{Spacecraft System with Gas Jets}

The controllability analysis for spacecraft system with gas jets is similar to that for the system with reaction wheels. Define conjugate momenta

$$
\mathbf{I}=\mathbf{I} \boldsymbol{\Omega} \quad \text { and } \quad \mathbf{p}=m \dot{\mathbf{x}} .
$$

The dynamical equations expressed as a canonical form on $T^{*}\left(S O(3) \times \mathbb{R}^{3}\right)$ are:

$$
\begin{aligned}
\dot{B} & =B \mathbf{I}^{-1} \mathbf{I} \mathbf{I} \\
\dot{B} \vec{\Pi} & =B \mathbf{I}^{-1} \Pi \Pi+B\left(\Pi \times \mathbf{I}^{-1} \Pi+\int_{\mathcal{B}} \frac{\mu\left(B^{\top} \mathbf{r} \times \bar{q}\right)}{|\mathbf{r}+B \bar{q}|^{3}} d m(\bar{q})\right)+B \hat{\tau} \\
\dot{\mathbf{r}} & =\frac{\mathbf{p}}{m} \\
\dot{\mathbf{p}} & =-\int_{B} \frac{\mu(\mathbf{r}+B \bar{q})}{|\mathbf{r}+B \bar{q}|^{3}} d m(\bar{q}) .
\end{aligned}
$$

Theorem 4 can be applied to prove the controllability of system (20). To this end, we first consider the Hamiltonian structure of the drift vector field of (20). By setting $\tau=0$, system (20) is a Hamiltonian system with the following Hamiltonian,

$$
H(B, B \hat{\mathbf{I}}, \mathbf{r}, \mathbf{p})=\frac{1}{2}<\mathrm{II}, \mathrm{III}>+\frac{1}{2} \frac{\mathbf{p}^{2}}{m}-\int_{\mathbf{B}} \frac{\mu}{|\mathbf{r}+B \bar{q}|} d m(\bar{q}) .
$$

Now, we clieck the Lie algebra rank condition. By choosing the control feedback

$$
\tau=-\mathbf{\Pi} \times \mathbf{I}^{-1} \mathbf{I}-\int_{\mathcal{B}} \frac{\mu\left(B^{\top} \mathbf{r} \times \bar{q}\right)}{|\mathbf{r}+\bar{B} \bar{q}|^{3}} d m(\bar{q})+\mathbf{u},
$$

system (20) can be transformed into the system (15) with II, replaced by $\bar{I}$, and $\mathrm{J}$ replaced by $\mathbf{I}$. Accordingly, the verification of Lie algebra rank condition is exactly the same as in the previous subsection. Therefore, we condition is exactly the san.

Theorem 6 Suppose the spacecraft system (20) with three-axes gas jets, i.e., the effects of gas jets are on three independent axes. Suppose also that $\mathbf{r}$ is finite and sufficiently large comparing to the size of the spacecrafi. Then the spacecraft system is controllable if the spacecraft has three distinct prin

$$
\int_{\mathcal{B}} \dot{q}^{\top}\left(-1+5 \mathbf{b}_{i} \mathbf{b}_{i}^{\top}\right) \bar{q} \bar{q}^{\top} d m(\bar{q}) \quad \text { V } \mathbf{b}_{i}, \forall i=1,2,3 .
$$

The above two Theorems 5 and 4 essentially tell us that the orbital motion can be controlled by suitably adjusting the attitude motion. In fact, we may define the center of gravity of the assembly to be, cf. [22], the vector $\mathbf{r}_{G}$ such that

$$
\frac{\mathbf{r}_{G}}{\left|\mathbf{r}_{G}\right|^{3}}=\int_{\mathbb{B}} \frac{\mathbf{r}+B \bar{q}}{|\mathbf{r}+B \bar{q}|^{3}} d m(\bar{q}) \text {. }
$$

Thus the center of gravity is the place where the total gravitational force acts on. It can be proved that while the center of mass is fixed in the assembly, the center of gravity varies with respect to different orientations of the assembly. From this observation, it is then natural that changing the attitude (relative to the center of mass) may lead to the cliange of the orbit.

\section{Conclusions}

In this paper, the equations of motion for spacecraft system with reaction wheels or with gas jets in a central gravitational field are concerned. For these dynamics, we are interested in investigating the possibility of controlling the orbit by attitude controllers. The formal definitions of the controllability and
weakly positive Poisson stability has been addressed. We showed that if the drift vector field of the system is weakly positively Poisson stable and the Lie algebra rank condition is satisfied, controllability can be concluded. For the above mechanical systems, their intrinsic Hamiltonian structures were the used to guarantee the weakly positive Poisson stability. On the other hand, the Lie algebra rank condition was verified with the aid of the techniques in computing of Lie brackets on second tangent bundle of $S O(3) \times \mathbb{R}^{3}$. The main results of this paper state that it is possible to change the orbit of main results of this paper state that it is possible to change the orbit of the spacecraft by controlling the attitude. The observation of such attitudespacecraft system but also may lead to new teclinologies in spacecraft control.

\section{References}

[1] R. Abraham and J.E. Marsden. Foundations of Mechanics. Benjamin/Cununings, 2nd edition, 1978.

[2] D.V. Anosov, I.U. Bronshtein, S.K. Aranson, and V.Z. Grines. Dynamical Systems I: Smooth Dynamical Systems. Encyclopaedia of Math. Sciences. Springer-Verlag, Berlin Heidelberg, 1988.

[3] V.I. Arnold, V.V. Kozlov, and A.I. Neihstadt. Dynamical Systems III. Encyclopaedia of Math. Sciences. Springer-Verlag, Berlin Heidelberg, 1988.

[4] B. Bommard. "Contrôlabilité des systèmes noulinéaires". C. R. Acad, Sci, Paris, 292:535-537, 1981

[5] R.W. Brockett, R.S. Millmalu, and H.J. Sussmam, editors. Differential Geometric Control Theory. Birkläuser, 1983.

[6] P. Brunovsky. Local Controllability of Odd Systems. Mathematical Control Theory Vol. 1. Banadi Center Publications, Warsaw, 1976.

[7] J.L. Casti. Nonlinear System Theary. Academic Press, 1985.

[8] P.E. Crouch. "Spacecraft attitude control and stabilization". IEEE Tran. Automatic Control, 29(4):321-331, 1984.

[9] P.E. Crouch and C.I. Byrnes. "Local accessibility, local reachability, and representations of compact groups". Math. Systems Theory, 19:43-65, 1986

[10] R. Hermann and A.J. Krener. "Nonlinear controllability and observability". IEEE Trans. Automatic Contral, 22(5):728-740, 1977.

[11] H. Hernes. "Control system which generate decomposable Lie algebra". J. Diff. Eqns., 44:166-187, 1982 .

[12] V. Jurdjevic and I. Kupka. "Control systems subordinated to a group action: Accessibility". J. of Differential Equations, 39:186-211, 1981.

[13] V. Jurdjevic and J.P. Quimn. "Controllability and stabibty". J. of Differential Equalions, 28:381-389, 1978.

[14] A.J. Krener. "A generalization of chow's theoresu and bang-bang theorem for nonlinear control problems". SIAM J. Control and Optimization, 12:43-52, 1974.

[15] A.J. Krener. " $\left(A d_{f, g}\right),\left(a d_{f, g}\right)$ and locally $\left(a d_{f, g}\right)$ invariant and controllability distributions". SIAM 'J. Control and Optimization, 23:523-549, 1985.

[16] E.B. Lee and L. Markus. Foundation of Optimal Control Theory. John Wiley, New York, 1976

[17] K.-Y. Lian. Dynamics and Control of Aircraft and Spacecrafi Systems. PhD thesis, National Taiwan University, Taiwan, 1993.

[18] K.-Y. Lian, L.-S. Wang, and L.-C. Fu. "Globsal attitude representation and its Lie bracket". In Proce of the 1993 American Conirol Conference, pages 425-420, 1993.

[19] C. Lobry. "Controllability of nonlinear systems on conpact manifolds". SIAM J. Control, 12(1):1-4, 1974 .

[20] A.R. Moller. Nonlinear Systems Vol. II, Applications to Bilinear Control. Prentice Hall, 1991.

[21] H. Nijmeijer and A.J. van der Schaft. Nonlinear Dynamical Control Systems. Springer-Verlag, 1990.

[22] F.P.J. Rimratt. Introductory Attitude Dynamics. Springer-Verlag, New York, 1989

[23] H.J. Sussmanu. "A general theorem on local controllability". SIAM J. Control and Optimization, 25(1):158-194, 1987.

[24] H.J. Sussmamn, editor. Nonlinear Controllability and Optimal Control. Marcel Dekker, New York, 1990. [25] H.J. Sussmain and V. Jurdjevic. "Controllability of nonlinear system". Journal of
Diff. Eqns., 12:313-329, 1972.

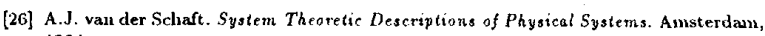

[27] F. Verlulst. Nonlinear Differential Equations and Dynamical Systems. SpringerVerlag, 1990.

[28] L.-S. Waig. Geometry, Dynamics and Control of Coupled Systems. PhD thesis, University of Maryland, 1990 .

[29] L.-S. Wang, P. S. Krishnaprasad, and J. H. Maddocks. "Hamiltonian dynamics of a rigid body in a cestral gravitational field". Celestial Mechanics and Dynamical Astronomy, 50:349-386, 1991.

[30] L.-S. Wang, K.-Y. Lian, and P.-T. Chen. "Steady motions of gyrostat satellites and their stability". submited.

[31] L.-S. Wang, J. H. Maddocks, and P. S. Krishnaprasad, "Steadly rigid-body motions in a central gravitational field". The Journal of the Astronautical Sciences, 40:449-478, 1992. [32] J. R. Wertz, editor. Spacecraft Attitude Determination and Control. Kluwer Academic
Publishers, 1978 .

[33] W.E. Wiesel. Spacefight Dynamics. McGraw-Hill, New York, 1989. 